\title{
Wave-vector dependence of spin and density multipole excitations in quantum dots
}

\author{
Manuel Barranco, ${ }^{*}$ Leonardo Colletti, and Enrico Lipparini \\ Dipartimento di Fisica, Università di Trento, and INFM sezione di Trento, I-38050 Povo, Italy \\ Agustí Emperador and Martí Pi \\ Departament ECM, Facultat de Física, Universitat de Barcelona, E-08028 Barcelona, Spain \\ Llorenç Serra \\ Departament de Física, Universitat de les Illes Balears, E-07071 Palma de Mallorca, Spain
}

(Received 21 July 1999; revised manuscript received 1 October 1999)

\begin{abstract}
We have employed time-dependent local-spin density-functional theory to analyze the multipole spin and charge density excitations in GaAs- $\mathrm{Al}_{x} \mathrm{Ga}_{1-x} \mathrm{As}$ quantum dots. The on-plane transferred momentum degree of freedom has been taken into account, and the wave-vector dependence of the excitations is discussed. In agreement with previous experiments, we have found that the energies of these modes do not depend on the transferred wave vector, although their intensities do. Comparison with a recent resonant Raman scattering experiment [C. Schüller et al., Phys. Rev. Lett. 80, 2673 (1998)] is made. This allows us to identify the angular momentum of several of the observed modes as well as to reproduce their energies.
\end{abstract}

\section{INTRODUCTION}

The characteristic single particle and collective excitations of typical quantum dots (QD) are known to lie in the far-infrared (FIR) energy region, i.e., they have energies that, depending on the size of the dot, span the range from a few tens of $\mathrm{meV}$ to a fraction of meV. Experimental information about FIR spectra was first obtained from photon absorption experiments on $\mathrm{InSb}$ and on GaAs quantum dots. ${ }^{1,2}$ Since the confining potential for small dots is parabolic to a good approximation, and in the FIR regime the dipole approximation works well, the absorption spectrum is rather insensitive to the number of electrons in the dot, measuring to a large extent only the center-of-mass excitations, which at nonzero magnetic fields $(B)$ correspond to the two allowed dipole transitions arising from each of the two possible circular polarizations of the absorbed light. Two limitations of the absorption process, namely that it is dominated by the $L=1$ multipole of the incoming electromagnetic wave, and its insensitivity to the electronic spin degree of freedom, have motivated that theorists have been mostly concerned with the study of dipole charge density excitations (CDE). Yet, higher multipolarity CDE's have been discussed using a classical model, ${ }^{3}$ a Hartree-random phase approximation method, ${ }^{4}$ a classical hydrodynamical model, ${ }^{5}$ and an equation of motion method. ${ }^{6}$ Quadrupole $L=2 \mathrm{CDE}$ 's have also been addressed for the quantum-dot helium. ${ }^{7}$

The situation is changing with the use of inelastic light scattering to study QD excitations. This experimental technique is nowadays recognized as one of the more powerful tools to study the elementary excitations of low-dimensional electronic nanostructures, ${ }^{8-13}$ and it is contributing to a deeper understanding of the two-dimensional electron gas $^{14-18}$ (2DEG). Using polarization selection rules, it allows us to disentangle CDE from spin density (SDE) and singleparticle excitations (SPE), and to observe them all in the same sample. Moreover, it offers the possibility of studying the wave-vector dispersion dependence of the excitations.

Measurements of Raman scattering on high-quality GaAs- $\mathrm{Al}_{x} \mathrm{Ga}_{1-x}$ As quantum dots have been reported. ${ }^{11}$ What makes this experiment especially appealing is that sharp spin and charge density excitations have been measured in conventional backwards geometry as a function of the applied magnetic field $B$ and of the transferred lateral wave vector $q$. Previous studies were carried out at zero magnetic field, 8,10 or the experimental conditions were such that the spectra did not show a wave-vector conservation nor a clear polarization dependence, ${ }^{9}$ hence it was not possible to resolve SDE and CDE from SPE, nor to record the spectra at predetermined $q$ values. We attempt here a theoretical interpretation of these results based on the time-dependent local-spin densityfunctional theory (TDLSDFT), addressing the description of high multipolarity spin and charge density modes of a QD, and incorporating in a realistic way the on-plane wave-vector dependence of these collective excitations. A recent calculation by Steinebach et al. ${ }^{19}$ has addressed the Raman scattering in small quantum dots at zero-magnetic field, including valence-band as well as multipole and momentum transfer effects. However, perfect spin degeneracy (paramagnetism) of the conduction electron states was imposed and correlation potentials were neglected. We explore here the $B$-dependence in general TDLSDFT but, since we are restricted to the conduction electron set, our results would correspond to off-resonance Raman peaks, with laser energies above the valence-conduction gap.

\section{TDLSDFT DESCRIPTION OF COLLECTIVE MODES IN QD}

The dipole longitudinal response of quantum dots has been recently addressed in detail. ${ }^{20,21}$ We sketch here how the method can be generalized to deal with other multipolarities and the wave-vector degree of freedom.

The first task is to obtain the ground state (gs) of the dot 
solving the appropriate Kohn-Sham (KS) equations. The exchange-correlation energy density $\mathcal{E}_{x c}(n, m)$, where $n$ is the electron density and $m$ the spin magnetization, constitutes a key ingredient of the method. It has been constructed from the results of Ref. 22 on the nonpolarized and fully polarized 2DEG using the two-dimensional von Barth and Hedin prescription to interpolate between both regimes. ${ }^{23}$

Once the KS gs has been worked out, we have determined the induced densities originated by an external excitation field employing linear-response theory. For independent electrons in the KS mean field, the variation $\delta n_{\sigma}^{(0)}$ induced in the spin density $n_{\sigma}(\sigma \equiv \uparrow, \downarrow)$ by an external spin-dependent field $F$, whose nontemporal dependence we denote as $F$ $=\Sigma_{\sigma} f_{\sigma}(\vec{r})|\sigma\rangle\langle\sigma|$, can be written as ${ }^{24}$

$$
\delta n_{\sigma}^{(0)}(\vec{r}, \omega)=\sum_{\sigma^{\prime}} \int d \vec{r}^{\prime} \chi_{\sigma \sigma^{\prime}}^{(0)}\left(\vec{r}, \vec{r}^{\prime} ; \omega\right) f_{\sigma^{\prime}}\left(\vec{r}^{\prime}\right)
$$

where $\chi_{\sigma \sigma^{\prime}}^{(0)}$ is the KS spin density correlation function. In this limit, the frequency $\omega$ corresponds to the harmonic time dependence of the external field $F$ and of the induced $\delta n_{\sigma}^{(0)}$. Equation (1) is a $2 \times 2$ matrix equation in the two-component Pauli space. In longitudinal response theory, $F$ is diagonal in this space, and its diagonal components are written as a vector $F \equiv\left(\begin{array}{l}f_{\uparrow} \\ f_{\downarrow}\end{array}\right)$. We consider first the external $L$-pole fields

$$
F_{ \pm L}^{(n)}=r^{|L|} e^{ \pm i L \theta}\left(\begin{array}{l}
1 \\
1
\end{array}\right) \text { and } F_{ \pm L}^{(m)}=r^{|L|} e^{ \pm i L \theta}\left(\begin{array}{c}
1 \\
-1
\end{array}\right)
$$

which cause, respectively, the charge and spin density $L$ modes. For the monopole $L=0$ mode, these fields are simply taken proportional to $r^{2}$ (see below). To distinguish the induced densities in each excitation channel they will be labeled with an additional superscript as $\delta n_{\sigma}^{(0, n)}$ or $\delta n_{\sigma}^{(0, m)}$.

The TDLSDFT induced densities are obtained from the integral equations

$$
\begin{aligned}
\delta n_{\sigma}^{(A)}(\vec{r}, \omega)= & \delta n_{\sigma}^{(0, A)}(\vec{r}, \omega)+\sum_{\sigma_{1} \sigma_{2}} \int d \vec{r}_{1} d \vec{r}_{2} \chi_{\sigma \sigma_{1}}^{(0)}\left(\vec{r}_{,} \vec{r}_{1} ; \omega\right) \\
& \times K_{\sigma_{1} \sigma_{2}}\left(\vec{r}_{1}, \vec{r}_{2}\right) \delta n_{\sigma_{2}}^{(A)}\left(\vec{r}_{2}, \omega\right)
\end{aligned}
$$

where either $A=n$ or $A=m$, and the kernel $K_{\sigma \sigma^{\prime}}\left(\vec{r}, \vec{r}^{\prime}\right)$ is the electron-hole interaction.

Equation (3) has been solved as a generalized matrix equation in coordinate space. Taking into account angular decompositions of $\chi_{\sigma \sigma^{\prime}}$ and $K_{\sigma \sigma^{\prime}}$ of the kind $K_{\sigma \sigma^{\prime}}\left(\vec{r}, \vec{r}^{\prime}\right)$ $=\Sigma_{l} K_{\sigma \sigma^{\prime}}^{(l)}\left(r, r^{\prime}\right) e^{i l\left(\theta-\theta^{\prime}\right)}$, it is enough to solve them for each multipole separately because only modes with $l= \pm L$ couple to the external $L$-pole field. One has

$$
\begin{aligned}
K_{\sigma \sigma^{\prime}}^{(l)}\left(r, r^{\prime}\right)= & \frac{2}{\pi^{3 / 2}} \frac{\Gamma(|l|+1 / 2)}{\Gamma(|l|+1)} \frac{r_{<}^{|l|}}{r_{>}^{|l|+1}} K_{|l|}\left(\frac{r_{<}}{r_{>}}\right) \\
& +\left.\frac{\partial^{2} \mathcal{E}_{x c}(n, m)}{\partial n_{\sigma} \partial n_{\sigma^{\prime}}}\right|_{g s} \frac{\delta\left(r-r^{\prime}\right)}{2 \pi r}
\end{aligned}
$$

where $K_{n}(x)$ is given by the hypergeometric function ${ }^{25}$ $\pi / 2 F\left(1 / 2, n+1 / 2 ; n+1 ; x^{2}\right)$, and $r_{>}\left(r_{<}\right)$is the greater (smaller) of $r, r^{\prime}$.

For a polarized system having a nonzero magnetization in the gs, the $\pm L$ modes are not degenerate and give rise to two excitation branches with $\Delta L_{z}= \pm L$, where $L_{z}$ is the gs orbital angular momentum. The induced charge or magnetization densities corresponding to density and spin responses are given by $\delta n^{(A)}=\delta n_{\uparrow}^{(A)}+\delta n_{\downarrow}^{(A)}$ and $\delta m^{(A)}=\delta n_{\uparrow}^{(A)}$ $-\delta n_{\downarrow}^{(A)}$. From them, the dynamical polarizabilities in the density and spin channels are, respectively given by

$$
\begin{gathered}
\alpha_{n n}(L, \omega)=\int d r r^{|L|+1} \delta n^{(n)}(r) \\
\alpha_{m m}(L, \omega)=\int d r r^{|L|+1} \delta m^{(m)}(r) .
\end{gathered}
$$

For each $L$ value, taking into account both $\pm L$ possibilities we define $\alpha_{A A}^{(L)}(\omega) \equiv \alpha_{A A}(L, \omega)+\alpha_{A A}(-L, \omega)$. Their imaginary parts are proportional to the strength functions $S_{A A}^{(L)}(\omega)=\operatorname{Im}\left[\alpha_{A A}^{(L)}(\omega)\right] / \pi$. The peaks appearing in the strength functions are the CDE or SDE excited by the external field. Analogously, the peaks appearing in the strength function which results from using in the above equations the KS density variations $\delta n_{\sigma}^{(0, A)}$ instead of the correlated ones $\delta n_{\sigma}^{(A)}$, correspond to the SPE.

An analysis based on the use of the above multipole excitation operators $r^{L} e^{ \pm i L \theta}$ implies that no appreciable onplane momentum $\vec{q}$ is transferred to the system, i.e., $q \approx 0$. This will become apparent below. Even in this limit, some interesting features of the experimental spectra are reproduced. Moreover, it allows one to make contact with FIR photoabsorption spectroscopy. Yet, a more detailed analysis of Raman spectra calls for introducing the $q$ dependence in a realistic way. A first attempt has been made in Ref. 9, although the analysis of the measured Raman spectra was carried out using a Hartree model that cannot address the spin degree of freedom on the one hand, nor take into account the contribution of charge and spin density collective modes to the scattering cross section on the other hand.

Hamilton and $\mathrm{McWhorter}^{26}$ were the first in pointing out the important role played by spin density modes in the Raman scattering in GaAs. Their original formulation has been further elaborated by Blum, ${ }^{27}$ and more recently the inelastic charge and spin density scattering cross sections have been discussed in terms of the charge $S_{n n}(q, \omega)$ and spin $S_{m m}(q, \omega)$ strength functions ${ }^{28}$ (often called dynamic structure functions)

$$
\begin{gathered}
\frac{d^{2} \sigma^{C}}{d \omega_{s} d \Omega_{s}} \propto\left|\hat{\mathbf{e}}_{i} \cdot \hat{\mathbf{e}}_{s}\right|^{2} S_{n n}(q, \omega) \\
\frac{d^{2} \sigma^{S}}{d \omega_{s} d \Omega_{s}} \propto\left|\hat{\mathbf{e}}_{i} \times \hat{\mathbf{e}}_{s}\right|^{2} S_{m m}(q, \omega),
\end{gathered}
$$

where $\omega$ is the energy difference of the incoming and scattered photon $\omega_{i}-\omega_{s}$, and $\hat{\mathbf{e}}_{i, s}$ are the polarization vectors. 


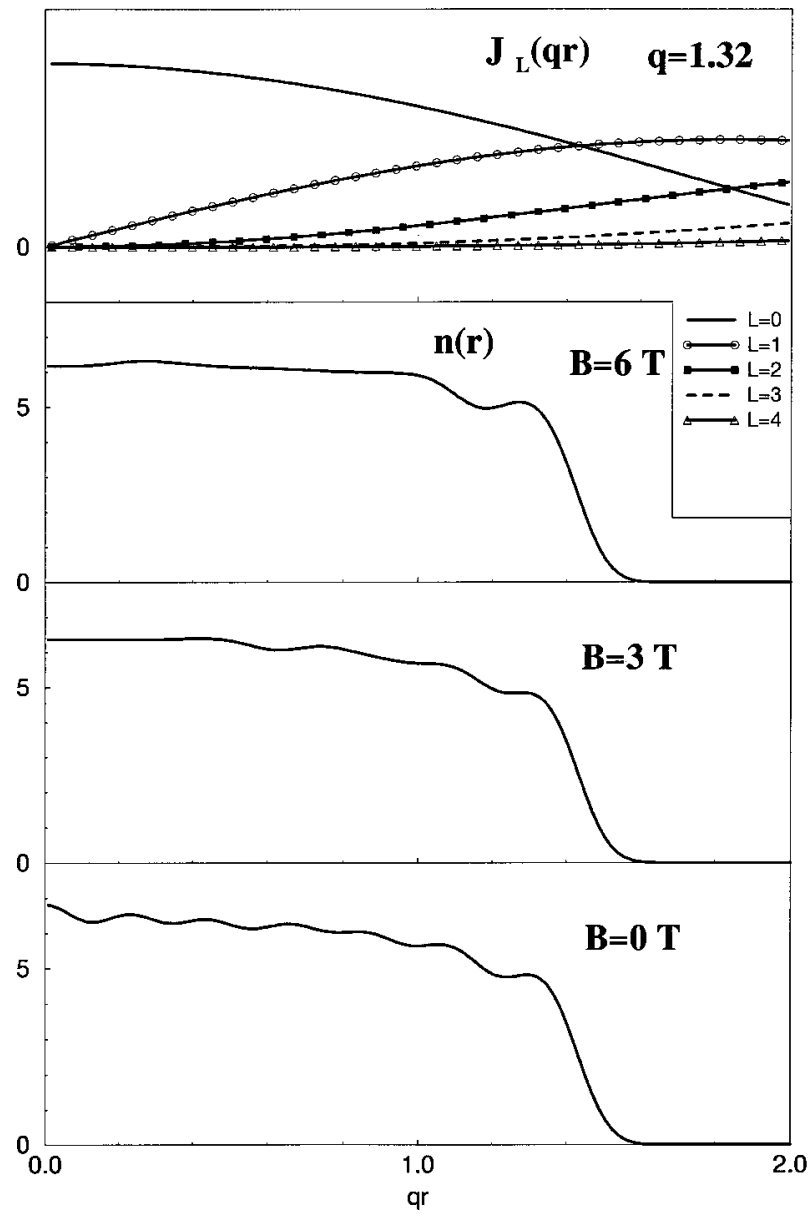

FIG. 1. Electron density of the $N=200$ dot (in units of $10^{11} \mathrm{~cm}^{-2}$ ) at $B=0,3$, and $6 \mathrm{~T}$. The dimensionless horizontal scale can be transformed into a more conventional one recalling that $q=1.32 \times 10^{5} \mathrm{~cm}^{-1}$. The value of $J_{L}(q r)$ for $L=0$ to 4 is also shown in the top panel for illustrative purposes.

We refer the reader to the review articles of Refs. 18 and 29 for a thorough discussion.

The above expressions are deceptively simple, but this is somehow misleading, as simplicity arises from the approximations made to arrive at them. ${ }^{27,28}$ Yet, they are often used to describe resonant Raman scattering in GaAs

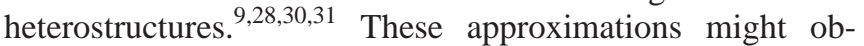
scure the comparison of the calculated modes with these detected by Raman spectroscopy. We believe, however, that rather than testing the TDLSDFT description of charge and spin density excitations, it may manifest the limitations of theoretical schemes based on Eq. (6) to analyze resonant Raman scattering. It is worth to mention the application made by Wendler et $a l .{ }^{32}$ to resonant Raman scattering in two electron quantum rings using a more general expression for the cross sections, of hopeless applicability to the $N$ $=200$ quantum dot described in Ref. 11, as well as the calculation by Steinebach et al. ${ }^{19}$ mentioned in the Introduction.

To obtain $S_{n n}(q, \omega)$ and $S_{m m}(q, \omega)$ within TDLSDFT, instead of considering the response to multipole operators, one has to consider the plane wave operator $e^{i \vec{q} r}$ involved in the inelastic scattering process. It is convenient to expand it into Bessel functions ${ }^{25}$

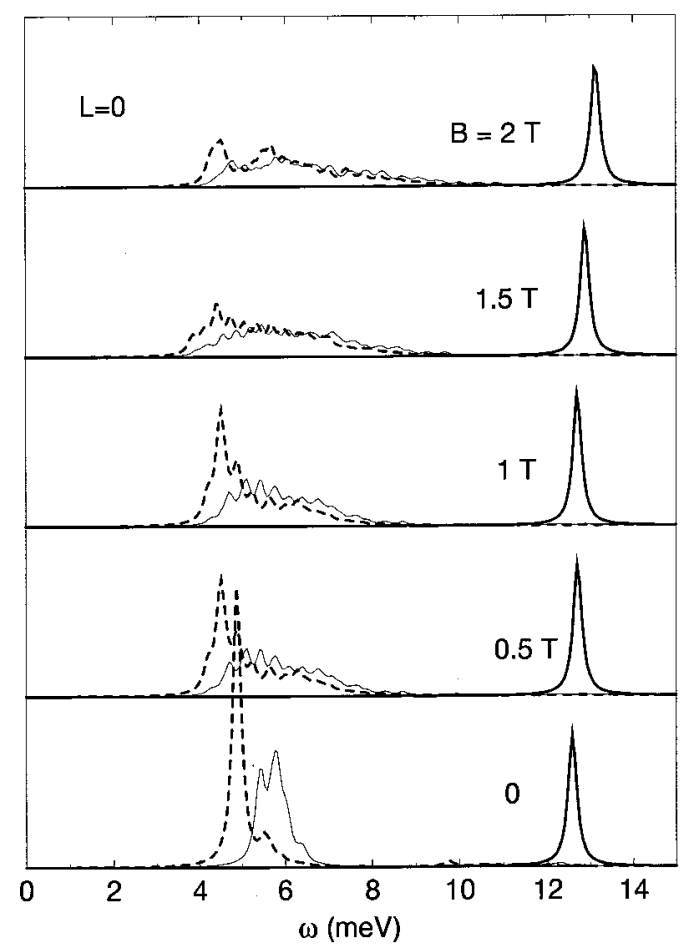

FIG. 2. Monopole strength function in arbitrary units as a function of energy. The thick-solid line represents the charge density strength, the dashed line the spin density strength, and the thin solid line the single-particle strength.

$$
\begin{aligned}
e^{i \vec{q} \vec{r}} & =\sum_{\forall L} i^{L} J_{L}(q r) e^{i L \theta} \\
& =J_{0}(q r)+\sum_{L>0} i^{L} J_{L}(q r)\left(e^{i L \theta}+e^{-i L \theta}\right) .
\end{aligned}
$$

Depending on the $q$ value, the number of terms in the expansion may be large, but the method is of direct applicability because the different $L$ terms in the expansion do not couple. Physically, it is also sound to make the expansion, since the experimental results display quite distinct peaks whose multipolar character can, in some cases, be identified even at a transferred momentum as large as $0.8 \times 10^{5} \mathrm{~cm}^{-1}$ (see Fig. 2 of Ref. 11). Moreover, in the small $q$ limit, the expansion of the Bessel functions leads to the multipole excitation operators we have previously considered. In particular, the $r^{2}$ operator used in the monopole case arises from the first nontrivial term in the expansion of $J_{0}(q r)$. An $r^{2}$ term is also present in the quadrupole case, this time multiplied by the angular operators $e^{ \pm 2 i \theta}$.

The TDLSDFT response to the plane wave operator can thus be obtained as in the multipole case substituting in Eq. (2) $r^{L}$ by $J_{L}(q r)$ and $r^{2}$ by $J_{0}(q r)$, and keeping as many terms in the expansion Eq. (7) as needed. A criterion to determine the number of terms to be considered is provided by the $f$-sum rule. ${ }^{21}$ For a given $q$ value, the $f$-sum rules of the plane wave operator and of each $L$ component in Eq. (7) read (in effective atomic units) 


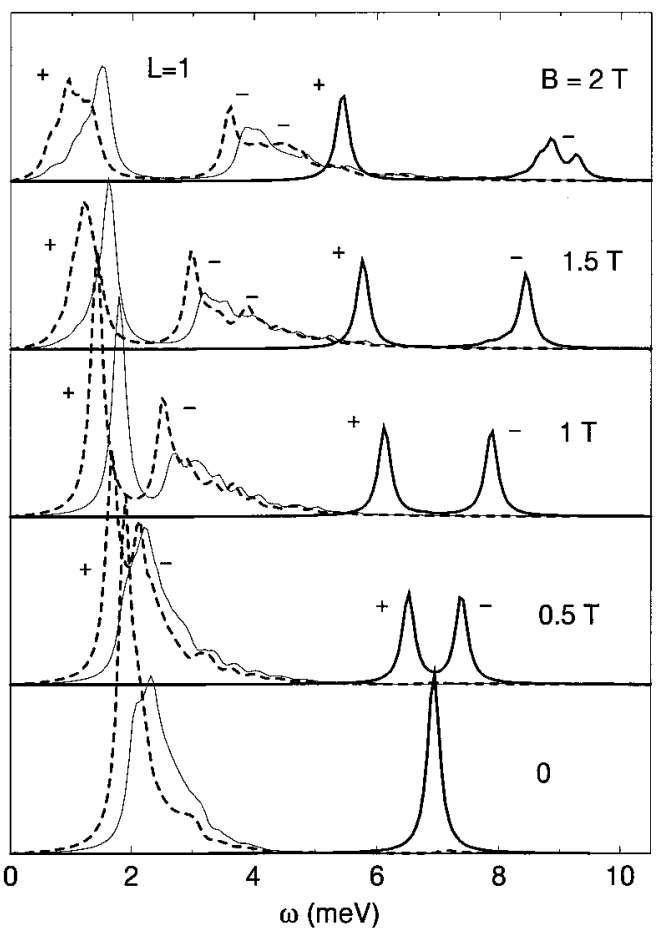

FIG. 3. Same as Fig. 2 for the dipole mode. The signs indicate the circular polarization of the more intense peaks, see Eq. (2).

$$
\begin{aligned}
m_{1}^{(n n)}\left[e^{i \vec{q} \cdot \vec{r}}\right] & =m_{1}^{(m m)}\left[e^{i \vec{q} \cdot \vec{r}}\right]=q^{2} \frac{N}{2}, \\
m_{1}^{(n n)}\left[J_{L}(q r) e^{i L \theta}\right] & =m_{1}^{(m m)}\left[J_{L}(q r) e^{i L \theta}\right] \\
= & \frac{1}{2} \int d \vec{r} n_{0}(\vec{r}) \\
& \times\left\{\left[\frac{d J_{L}(q r)}{d r}\right]^{2}+\frac{L^{2}}{r^{2}} J_{L}^{2}(q r)\right\},
\end{aligned}
$$

where $n_{0}(\vec{r})$ is the gs electron density. The maximum $L$ value in the expansion has been fixed so as to fulfill the plane-wave $f$-sum rule within $95 \%$ or better. As a further numerical test, the second Eq. (8) has been used to check the accuracy in the calculation of the strength functions multipole by multipole.

\section{RESULTS}

As a case of study, we present a theoretical interpretation of the results obtained in Ref. 11 for an $N=200$ electron quantum dot of radius $R=120 \mathrm{~nm}$ in $\mathrm{GaAs}_{-} \mathrm{Al}_{x} \mathrm{Ga}_{1-x} \mathrm{As}$. We have modeled the confining potential by the Coulomb potential created by a positively charged jellium disk of the same radius. ${ }^{33}$ The only free parameter in the calculation is the number of positive charges in the disk, which has been set to $N^{+}=404$ to reproduce as many spin and density modes as possible at $B=0$, with a special emphasis in the dipole SDE. We want to stress that this particular jellium disk plays no other role that creating a confining potential easy to generate and vary in a controlled way by simply changing $N^{+}$. The question of whether the system is charged

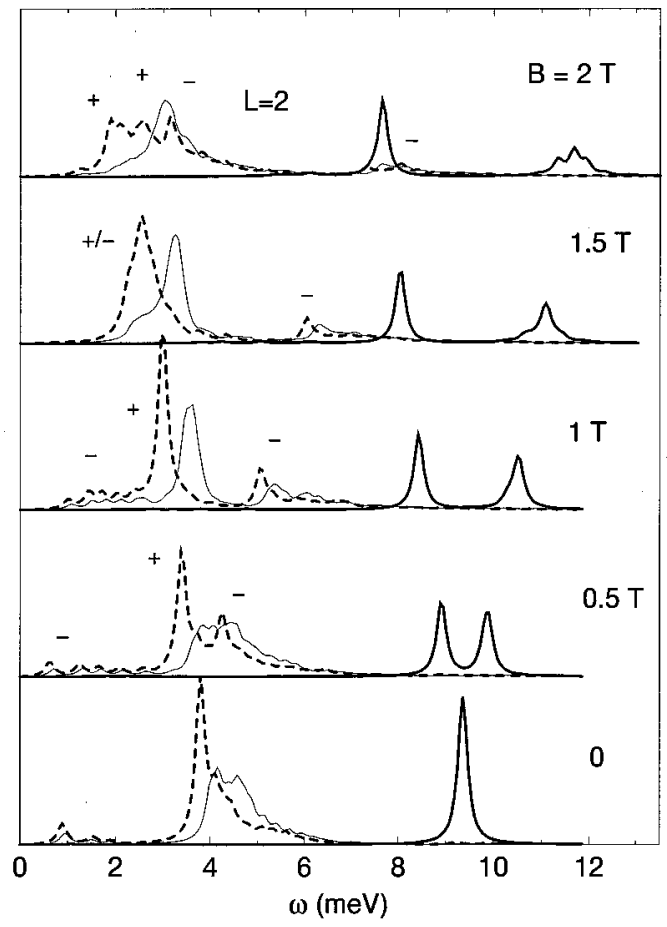

FIG. 4. Same as Fig. 3 for the quadrupole mode.

or not is misleading; after all, it could not be more "charged" than any $N$ electron dot confined by a parabolic potential. Image charges representing the gates and usually not considered in QD structure calculations will eventually make neutral the whole system. ${ }^{34}$ Discarding a parabolic potential because of the large number of electrons in the dot, other confining potentials ${ }^{9,35,36}$ and fitting parameter strategies might have been considered. However, a thorough testing of the confining potential for such a large dot would imply to obtain the charge and spin responses at $B=0$ for several multipoles. Obviously, this is a very demanding task. A more elaborated search could have improved the results we are going to discuss, which in some cases are not in full agreement with experiment. Figure 1 shows the electron densities at $B=0,3$, and $6 \mathrm{~T}$.

The choice of the spin dipole mode at $B=0$ as the experimental quantity to be better reproduced in the fit is motivated by the emphasis we want to put in the spin channel results, and because for this mode two distinct branches with positive and negative $B$ dispersions are seen in the experiment. The dipole $\mathrm{CDE}$ at $B=0$ would have been a more conventional choice, but unfortunately its experimental value has not been reported. ${ }^{10,11}$ It is worth it to point out that even if there seems to exist a common belief that CDE's are well understood, for multipolarities different from the thoroughly studied dipole mode this belief does not stem from having so far confronted theory with real experiments. It is still an open question how quantitative is the agreement between theory and experiment when several CDE's have to be simultaneously described for the same QD.

The range of $B$ values investigated in this work corresponds to filling factors larger than 3. Consequently, the use of other density functional approaches such as current density functional theory (CDFT) better suited at high magnetic fields ${ }^{33,37,38}$ can be avoided. For a discussion of the difficul- 


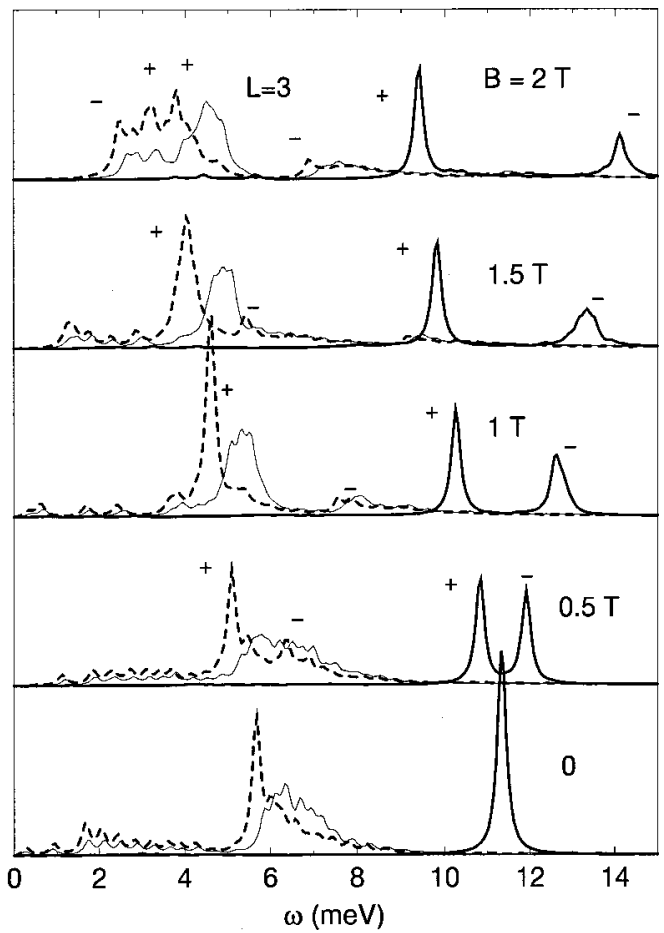

FIG. 5. Same as Fig. 3 for the octupole mode.

ties one has to face to obtain the longitudinal response within time-dependent CDFT, we refer the reader to Ref. 39.

\section{A. $q \approx 0$ results}

We first present the results obtained at $q \approx 0$. The interest in studying this limit lies in the experimental observation, 8,10 thoroughly discussed at $B=0$, that in QD's the energies of the excited modes do not depend on the transferred wave vector $q$. This is at variance with the situation in nanowires and in the 2DEG, constituting a clear signature of the "zero dimension' character of QD's. What changes with increasing $q$ is the total strength [see the first Eq. (8)], and how it is distributed among the different peaks. We shall discuss these matters in the next section.

Figures 2-5 represent the spin and charge strength functions for $L=0$ to 3 . In the $L \neq 0$ cases we have indicated with a $-(+)$ sign the excitations caused by the $+L(-L)$ component of the $F$ operators ${ }^{40}$ in Eq. (2). They correspond to the two possible circular polarizations of the light absorbed or emitted in the excitation or deexcitation process. We have found that the spin peaks are rather fragmented, especially in the monopole case. However, they still are collective modes, with energies redshifted from the single-particle ones due to the attractive character of the exchange-correlation vertex corrections.

We would like to draw the attention to the - type, lowenergy octupole SDE, which is seen in Fig. 5 to carry an appreciable strength at $\omega \sim 2.5 \mathrm{meV}$ for $B=2 \mathrm{~T}$. When a magnetic field is perpendicularly applied to a QD, it is well known that low-energy modes in the density channel are dipole edge CDE's arising from intraband transitions, while bulk interband transitions lie at higher energy. That may change with increasing $L$, and it is easy to see that this is indeed the case for SDE's. An inspection of the KS single

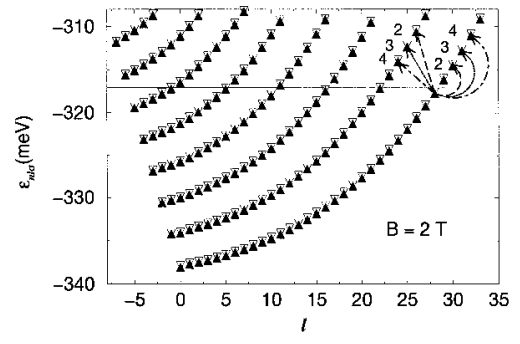

FIG. 6. Single-electron energies as a function of orbital angular momentum for $B=2$ T. Our choice of $B$ pointing towards $+z$ favors that single-particle states of negative angular momentum and upwards spin be occupied. To avoid dealing with single-particle angular momentum quantum numbers that are mostly negative, the angular dependence of the single-particle wave functions is written as $e^{-i l \theta}$ and hence, $l$ represents the orbital angular momentum changed of sign. The horizontal line represents the electron chemical potential. Full, upright triangles correspond to $\sigma=\uparrow$ states, and the empty, downright triangles to $\sigma=\downarrow$ states. Interband and intraband transitions with $\Delta l=2,3$, and 4 are represented to illustrate the energy crossing discussed in the text.

electron energies shown in Fig. 6 reveals that at high $L$ 's, interband electron-hole excitations are at lower energies than intraband ones. Since the electron-hole interaction is weak in the spin channel (only the exchange-correlation energy contributes to it), we have found that at $B=2 \mathrm{~T}$ the lowest energy octupole SDE is a mode built from interband electron-hole excitations. Still, one might consider it as an edge mode, as its existence is only possible because of the finite size of the system. When $B$ increases further, the spin density edge mode has again a + polarization. In the $L=3$, this happens at $B=3$ T. We have found that the low energy $\mathrm{CDE}$ is always a + type excitation, whereas the high energy CDE's are - type excitations arising from the corresponding component of $F^{(n)}$.

Figures 7 and 8 display the $B$ dispersion of the more intense CDE's and SDE's, respectively. The cyclotron frequency appears as a peak in the calculated SPE (KS) dipole response, and we have not plotted it in Fig. 7. The solid symbols represent the experimental data. ${ }^{11}$ We have connected with lines the more intense peaks obtained in the calculation of the strength, which displays some fragmentation, especially for high $L$ and $B$ values (see also Ref. 4). We recall that only for a pure parabolic confinement of frequency $\omega_{0}$ and for the $L=1$ mode in the dipole approximation, generalized Kohn's theorem ${ }^{41}$ ensures that CDE's are distributed according to the classical dispersion laws $\Omega$ $\pm \omega_{c} / 2$, with $\Omega^{2}=\omega_{0}^{2}+\omega_{c}^{2} / 4$ and $\omega_{c}$ being the cyclotron frequency. We also recall that the adiabatic TDLSDFT we are employing fulfills generalized Kohn's theorem. ${ }^{21}$

It can be seen from these figures that the experimental data are only partly explained, as not all the experimental modes are quantitatively described. In both spin and charge density channels, TDLSDFT reproduces the weak $B$ dependence of the $L=0$ mode found in the experiment at small $B$ values. Our calculation confirms the $L=0,1$, and 2 multipolarity assigned in the experiment to the lower SDE's, but cannot identify the origin of the higher SDE, whose signal is weak and broad, as mentioned in Ref. 11. We will see in the next section that including finite momentum transfer, as in actual experiments, does not greatly clarify the situation. 


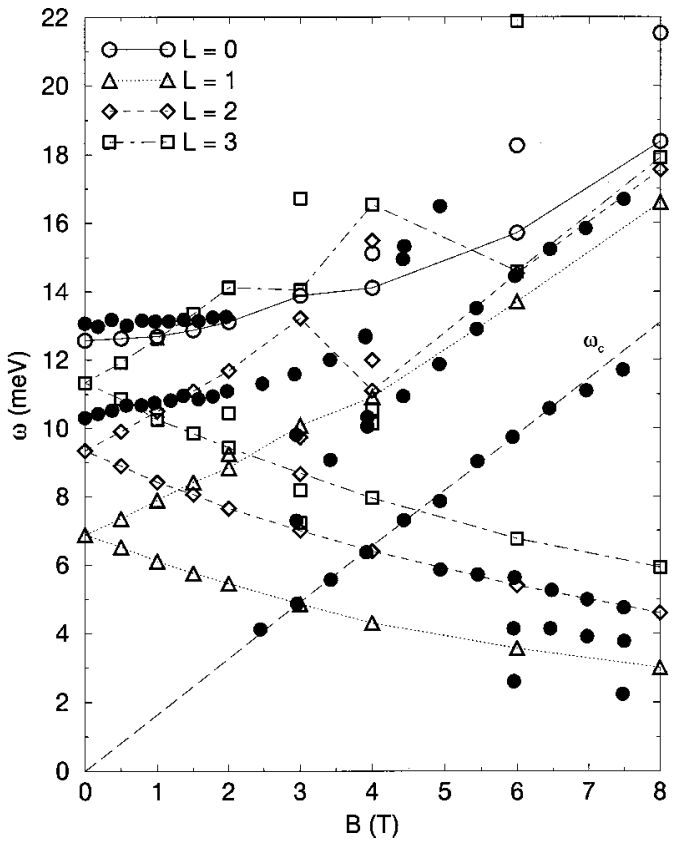

FIG. 7. Energies of the more intense CDE's as a function of $B$. The lines connect the more intense peaks corresponding to a given multipole, and the solid symbols represent the experimental data. ${ }^{11}$

At $B=0$, the energies of the $L>0$ spin density excitations follow the simple rule $E_{L} \sim L E_{1}$. We attribute this to the weakness of the electron-hole interaction in the spin channel. The prominent role played by the strong electron-hole interaction in the charge density channel causes that rule to fail for CDE's.

As a general trend, the strength carried by the positive $B$ dispersion branch corresponding to the high $L$ spin density excitations diminishes as $B$ increases. We have also found that the spin strength becomes more fragmented with increasing $L$, whereas bulk and edge magnetoplasmons associated with the $\pm L$ excitations are better defined modes.

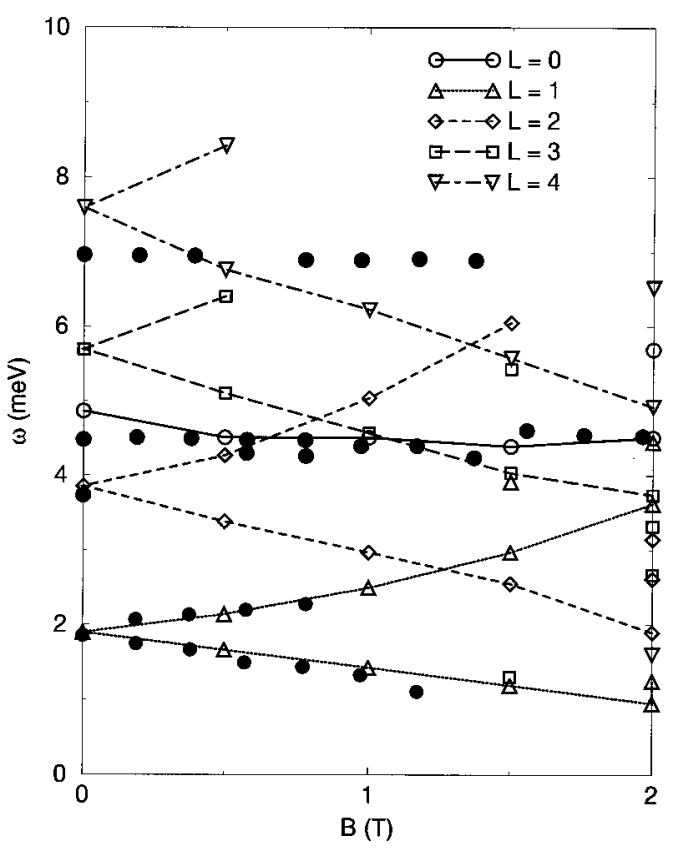

FIG. 8. Same as Fig. 7 for the more intense SDE's.
The positive $B$ dispersion branches of the CDE's reveal a complicated pattern at intermediate $B$ values, quite different from the expected classical one holding up to $B \sim 2-3 \mathrm{~T}$, but that however fits a large set of the experimental modes. The behavior of these branches has an interesting quantal origin, namely the formation of well-defined Landau bands for magnetic fields larger than a critical value. Above it, the more intense high energy collective peaks mostly arise from transitions between Landau bands whose index $M$ differs in one unit, $\Delta M=1$. Since these bands are made of many single electron states with different $l$ values and energies rather $l$ independent if $B$ is high enough, ${ }^{33}$ this explains the otherwise striking quasi $L$ degeneracy of the plasmon energies, only broken by finite size effects and the $L$ dependence of the electron-hole interaction. Other modes with $\Delta M=2$ build branches satellite of those formed by the more intense $L$ peaks, and are clearly seen in the calculation. Satellite branches of this kind appear even in the dipole case, ${ }^{2,42}$ and are a clear signature of nonparabolic confinement. ${ }^{4,521} \mathrm{We}$ will see below how these branches emerge at high $q$ and $B$ values.

In contradistinction with the positive $B$ dispersion branches of the CDE's, the negative $B$ dispersion ones do not manifest the quasi $L$ degeneracy. While interband electronhole excitations at the bulk of the dot are rather $L$ independent as we have just mentioned, the negative $B$ dispersion branches are built from intraband electron-hole excitations at the dot edge, and these are quite distinct for different $L$ values (see Fig. 6, and Fig. 5 of Ref. 33 for instance).

\section{B. Finite $q$ results}

The linear response to the multipole fields described before cannot tell what is the relative intensity of the different charge or spin density excitations. This limitation is circumvented using the plane wave operator for which $S_{n n}(q, \omega)$ and $S_{m m}(q, \omega)$ display the charge or spin density excitations with nonarbitrary relative intensity, allowing one to ascertain in each channel which $L$ modes are more probably excited at given $B$ and $q$ values. This is clearly seen in Figs. 9-14.

Figure 9 shows the CDE's and SDE's at $B=0$ for selected $q$ values used in Refs. 9 and 11 (we shall give $q$ in $10^{5} \mathrm{~cm}^{-1}$ ). Several interesting features show up in this figure. We see that for small $q$ values the dipole mode takes most of the strength, and that for the $q$ values employed in Ref. 11, the strength is exhausted by the modes with $L \leqslant 3$. Another interesting observation, in full agreement with experiments, is that the peaks have no appreciable wave-vector dispersion. ${ }^{8,10}$

For a given $L$, Fig. 9 also reveals the mechanism by which the strength evolves with increasing $q$. Up to the $q$ values of Ref. 11 only the lowest energy peak of each multipolarity is sizably excited, and with increasing $q$ strength is transferred from dipole to quadrupole, monopole and octupole, successively. For larger $q$ values, as those employed in Ref. 9, higher energy peaks of each multipolarity get predominantly excited. Conspicuous peaks corresponding to the second dipole and quadrupole modes, respectively, can be clearly seen at $q=5$ and $\omega \sim 16$ and $\sim 17 \mathrm{meV}$. The same happens at finite $B$ values, as it is shown in Fig. 10 for $B=1$ T.

Figures 11 and 12 show the evolution with $B$ of the spectra corresponding to the largest $q$ value of Ref. 11. Figures 


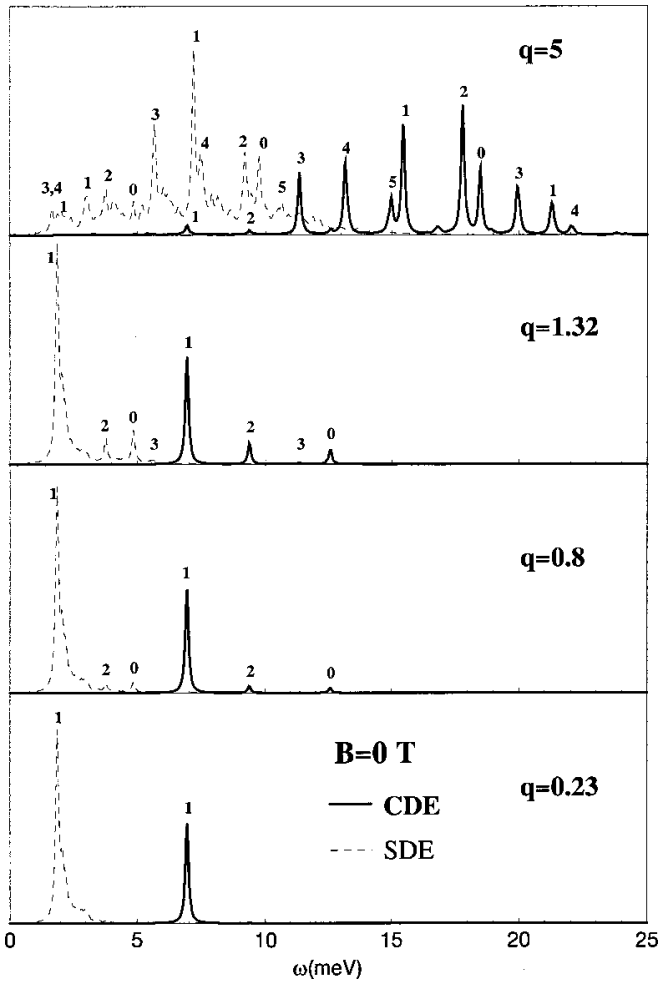

FIG. 9. $B=0$ charge (solid lines) and spin density (dashed lines) strengths in arbitrary units for $q=0.23,0.8,1.32$, and 5 $\times 10^{5} \mathrm{~cm}^{-1}$. The multipolarity of the main peaks is indicated.

13 and 14 show the same for the largest $q$ used in Ref. 9. As anticipated, Fig. 12 does not help identify the nature of the high-energy SDE detected in the experiment. However, the results at higher $q$ (Fig. 14) show at low $B$ a broad distribu-

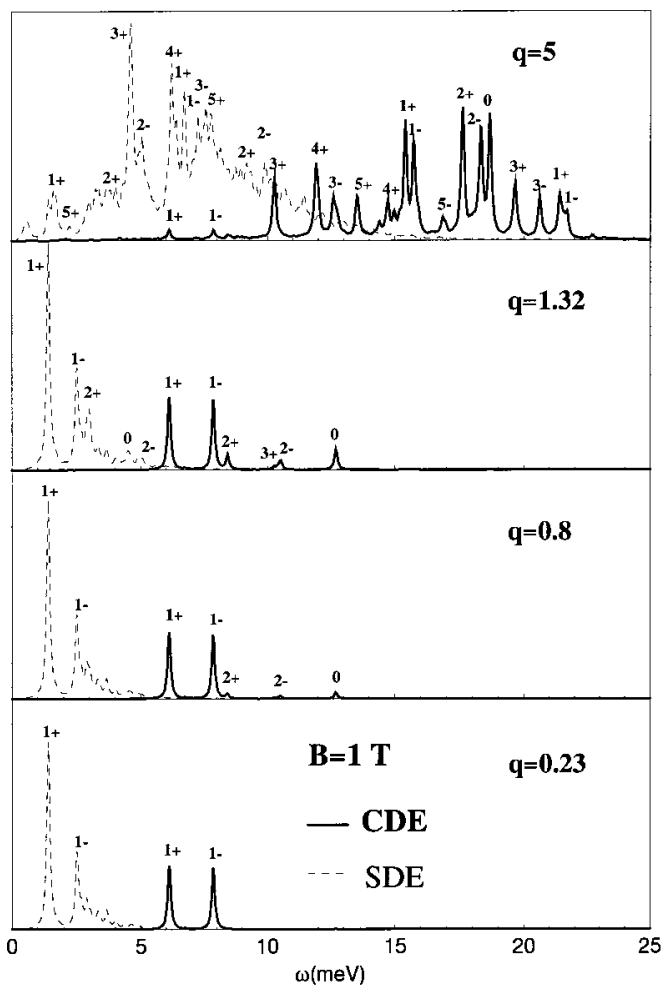

FIG. 10. Same as Fig. 9 for $B=1$ T.

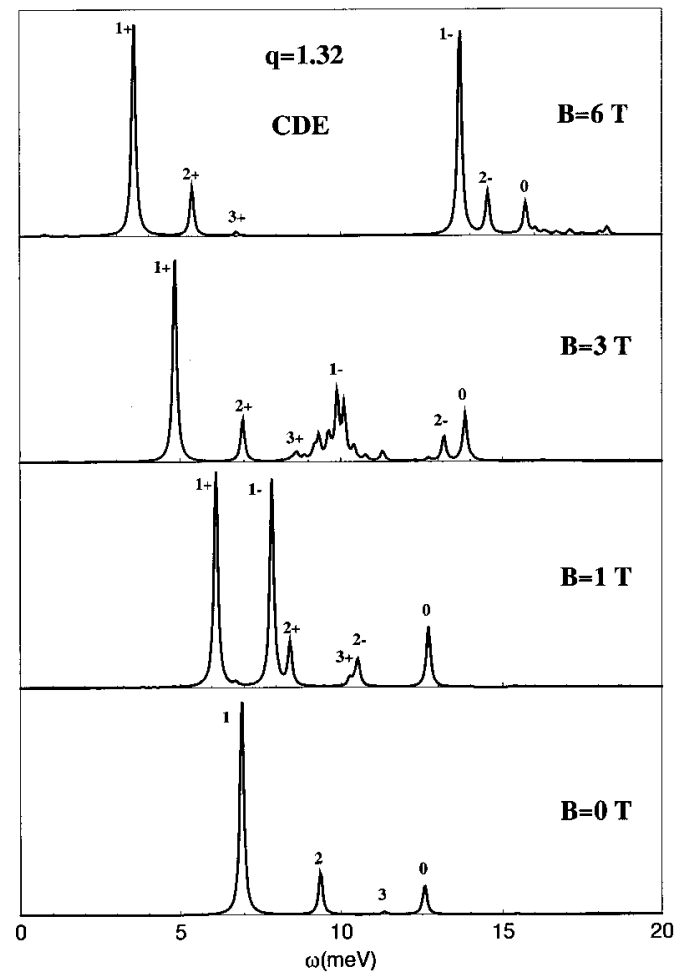

FIG. 11. Charge density strengths in arbitrary units for $q$ $=1.32 \times 10^{5} \mathrm{~cm}^{-1}$ and different $B$ values. The multipolarity and polarization of the main peaks is indicated.

tion of the SDE strength centered around the energy of that experimental mode. One is tempted to speculate that the higher SDE seen in the experiment is just the envelope corresponding to our higher $q$ spectrum, which is centered around the second dipole SDE. In this sense, it is worth to

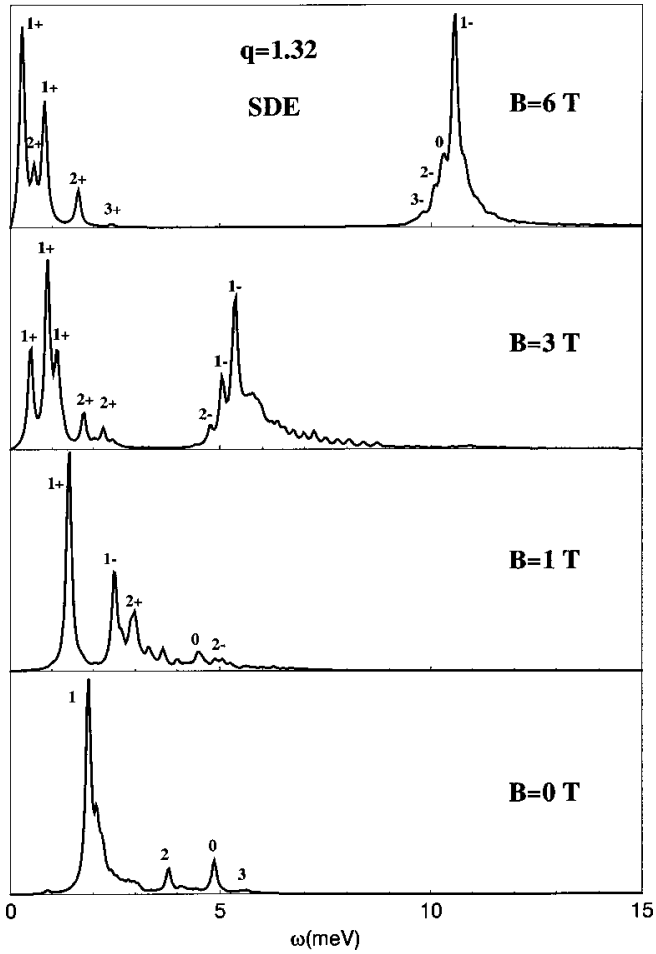

FIG. 12. Same as Fig. 11 for the spin density strength. 


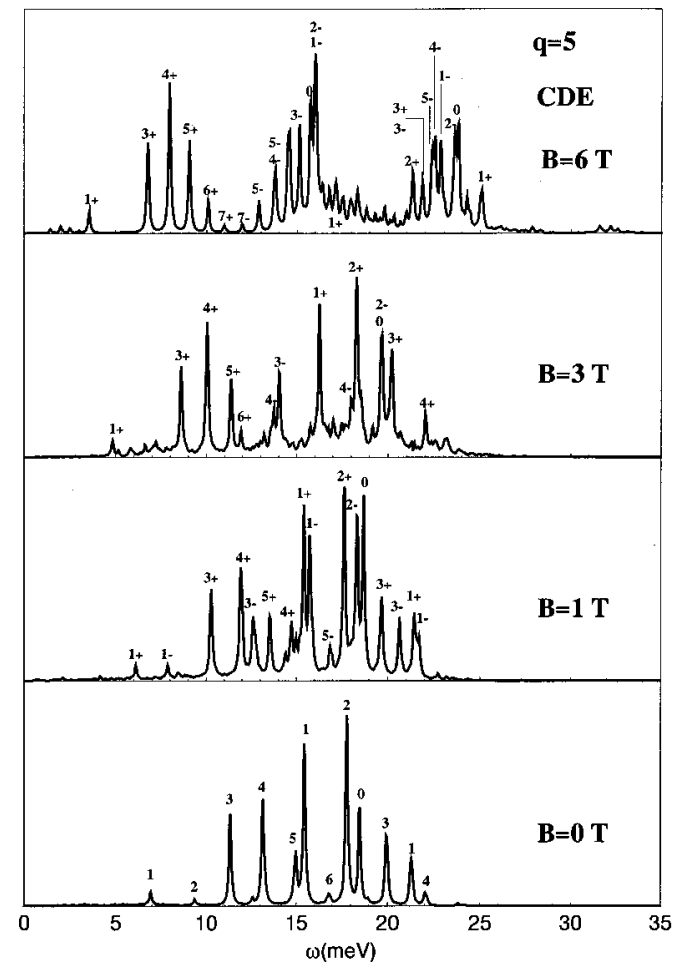

FIG. 13. Charge density strengths in arbitrary units for $q=5$ $\times 10^{5} \mathrm{~cm}^{-1}$ and different $B$ values. The multipolarity and polarization of the main peaks is indicated.

point out that our LSDA is essentially equivalent to a contact interaction in the spin channel, and thus it may underestimate finite momentum effects. Of course, a similar effect could also contribute to the broad features observed in the higher CDE's. The $B=6 \mathrm{~T}$ panels in Figs. 11 and 13 show that CDE's have a tendency to bundle, the energy spacing between bundles roughly being $\omega_{c}$. We have already discussed this effect at $q=0$.

Finally, we have used our results at $q=1.32$ to estimate the ratio $r=\left(\omega_{S P E}-\omega_{S D E}\right) /\left(\omega_{C D E}-\omega_{S P E}\right)$ for the more intense peaks. This ratio is a quantitative measure of the manyelectron interactions in the dot. ${ }^{10}$ At $B=0$ we have obtained $r \sim 0.11$, in good agreement with the experimental value. ${ }^{10}$ This ratio decreases with increasing $B$; we have found that $r \sim 0.08$ at $B=6 \mathrm{~T}$.

\section{SUMMARY}

In this paper we have thoroughly discussed spin and charge density modes of different multipolarity in GaAs- $\mathrm{Al}_{x} \mathrm{Ga}_{1-x}$ As quantum dots, as well as their wavevector dependence. This has allowed us to make a detailed comparison with experimental data obtained from resonant Raman scattering. In particular, our calculations reproduce the experimental finding that the excitation energies of the modes do not depend on the transferred wave-vector, although their intensities do. The ratio $\left(\omega_{S P E}-\omega_{S D E}\right) /\left(\omega_{C D E}\right.$ $\left.-\omega_{S P E}\right)$ is also reproduced.

We have been able to compare the energies of several spin and density modes arising in the same dot. After fitting the value of the spin density dipole mode at zero magnetic

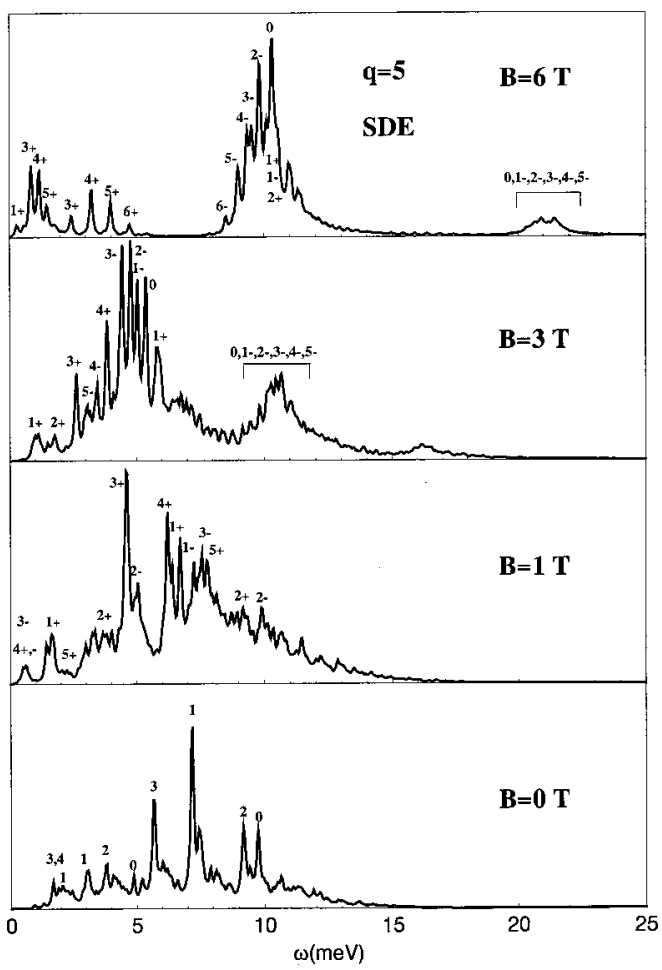

FIG. 14. Same as Fig. 13 for the spin density strength.

field, the energies of the spin density modes up to $L=2$ haven been quantitatively reproduced as a function of $B$.

The origin of the high energy spin density mode at $q$ $=1.32$ has not been elucidated by our calculations, although our results for larger $q$ 's predict a very broad distribution of strength centered around this experimental value. The analysis of the strength function at the experimental wave-vector $q$ seems to indicate that no appreciable strength is carried by modes with $L>3$, and that a broad structure consisting of peaks of different multipolarity $L \leqslant 3$ and polarization appears between 1 and $3 \mathrm{~T}$ (see the appropriate panels in Fig. 12).

The top panel of Fig. 1 gives a hint about the difficulty to properly describe high- $L$ modes with rather simple confining potential models. While for $L=0$ to 2 the excitation operator is probing the bulk region and part of the edge of the dot, for higher multipolarities it is only sensitive to its outermost edge structure. ${ }^{6}$ Obviously, for a large $N$ dot this region is very much influenced by the actual structure of the confining potential, and one should expect the larger disagreements between theory and experiment to appear for these modes.

In the charge density channel, the agreement between theory and experiment is more qualitative. At $B=0$ one of the measured CDE's is between our calculated $L=2$ and $L$ $=3$ modes and, as in the spin density channel, only the $L$ $=0$ mode is not appreciably dispersed with $B$. Yet, we have given an interpretation, and a fair quantitative description, of the peaks measured at intermediate $B$ values that lie between the $\omega_{c}$ and $2 \omega_{c}$ lines. As indicated, we have given more weight in the fitting procedure to reproducing the SDE's.

Finally, we would like to point out that in spite of the difficulties in interpreting resonant Raman scattering in terms of spin and density modes arising only from excitations of 
the conduction band electrons, several features of the spectra are well described within time-dependent local-spin densityfunctional theory. A more quantitative description of some aspects of the experimental spectra would require to take fully into account the underlying structure of the system beyond the simple, idealized semiconductor model currently used to describe quantum dots, and likely a more realistic confining potential in the case of high multipolarities.

\section{ACKNOWLEDGMENTS}

This work has been performed under Grant Nos. PB951249 and PB95-0492 from CICYT, Spain, and 1998SGR00011 from Generalitat of Catalunya. A.E. and M.B. (Ref. PR1997-0174) acknowledge support from the DGES (Spain).
*Permanent address: Departament d'Estructura i Constituents de la Matèria, Facultat de Física, Universitat de Barcelona, E-08028 Barcelona, Spain.

${ }^{1}$ Ch. Sikorski and U. Merkt, Phys. Rev. Lett. 62, 2164 (1989).

${ }^{2}$ T. Demel, D. Heitmann, P. Grambow, and K. Ploog, Phys. Rev. Lett. 64, 788 (1990).

${ }^{3}$ V. Shikin, S. Nazin, D. Heitmann, and T. Demel, Phys. Rev. B 43, 11903 (1991).

${ }^{4}$ V. Gudmundsson and R.R. Gerhardts, Phys. Rev. B 43, 12098 (1991).

${ }^{5}$ Z.L. Ye and E. Zaremba, Phys. Rev. B 50, 17217 (1994).

${ }^{6}$ E. Lipparini, N. Barberan, M. Barranco, M. Pi, and Ll. Serra, Phys. Rev. B 56, 12375 (1997).

${ }^{7}$ M. Wagner, A.V. Chaplik, and U. Merkt, Phys. Rev. B 51, 13817 (1995).

${ }^{8}$ R. Strenz, U. Bockelmann, F. Hirler, G. Abstreiter, G. Böhm, and G. Weimann, Phys. Rev. Lett. 73, 3022 (1994).

${ }^{9}$ D.J. Lockwood, P. Hawrylak, P.D. Wang, C.M. Sotomayor Torres, A. Pinczuk, and B.S. Dennis, Phys. Rev. Lett. 77, 354 (1996).

${ }^{10}$ C. Schüller, G. Biese, K. Keller, C. Steinebach, D. Heitmann, P. Grambow, and K. Eberl, Phys. Rev. B 54, R17 304 (1996)

${ }^{11}$ C. Schüller, K. Keller, G. Biese, E. Ulrichs, L. Rolf, C. Steinebach, D. Heitmann, and K. Eberl, Phys. Rev. Lett. 80, 2673 (1998).

${ }^{12}$ A.R. Goni, A. Pinczuk, J.S. Weiner, J.M. Calleja, B.S. Dennis, L.N. Pfeiffer, and K.W. West, Phys. Rev. Lett. 67, 3298 (1991).

${ }^{13}$ A. Schmeller, A.R. Goni, A. Pinczuk, J.S. Weiner, J.M. Calleja, B.S. Dennis, L.N. Pfeiffer, and K.W. West, Phys. Rev. B 49, 14 778 (1994).

${ }^{14}$ M.A. Eriksson, A. Pinczuk, B.S. Dennis, S.H. Simon, L.N. Pfeiffer, and K.W. West, Phys. Rev. Lett. 82, 2163 (1999).

${ }^{15}$ V. Pellegrini, A. Pinczuk, B.S. Dennis, A.S. Plaut, L.N. Pfeiffer, and K.W. West, Phys. Rev. Lett. 78, 310 (1997).

${ }^{16}$ A. Pinczuk, D. Heiman, S. Schmitt-Rink, C. Kallin, B.S. Dennis, L.N. Pfeiffer, and K.W. West, in Light Scattering in Semiconductor Structures and Superlattices, edited by D.J. Lockwood and J.F. Young (Plenum Press, New York, 1991), p. 571.

${ }^{17}$ A. Pinczuk and G. Abstreiter, in Light Scattering in Solids V, edited by M. Cardona and G. Guntherodt, Topics in Applied Physics Vol. 66 (Springer-Verlag, Berlin, 1989), p. 153.

${ }^{18}$ G. Abstreiter, M. Cardona, and A. Pinczuk, in Light Scattering in Solids IV, edited by M. Cardona and G. Guntherodt, Topics in Applied Physics Vol. 54 (Springer-Verlag, Berlin, 1984), p. 5.

${ }^{19}$ C. Steinebach, C. Schüller, and D. Heitmann, Phys. Rev. B 59, 10 240 (1999)

${ }^{20}$ L1. Serra, M. Barranco, A. Emperador, M. Pi, and E. Lipparini, cond-mat/9806104, Eur. Phys. J. D (to be published).
${ }^{21}$ L1. Serra, M. Barranco, A. Emperador, M. Pi, and E. Lipparini, Phys. Rev. B 59, 15290 (1999).

${ }^{22}$ B. Tanatar and D.M. Ceperley, Phys. Rev. B 39, 5005 (1989).

${ }^{23}$ U. von Barth and L. Hedin, J. Phys. C 5, 1629 (1972).

${ }^{24}$ A.R. Williams and U. von Barth, in Theory of the Inhomogeneous Electron Gas, edited by S. Lundqvist and N. March (Plenum, New York, 1983), p. 231.

${ }^{25}$ I.S. Gradshteyn and I.M. Ryzhik, Table of Integrals, Series and Products (Academic, New York, 1980).

${ }^{26}$ D.C. Hamilton and A.L. McWhorter, in Light Scattering Spectra of Solids, edited by G.B. Wright (Springer, Berlin, 1969), p. 309.

${ }^{27}$ F.A. Blum, Phys. Rev. B 1, 1125 (1970).

${ }^{28}$ S. Katayama and T. Ando, J. Phys. Soc. Jpn. 54, 1615 (1985).

${ }^{29}$ M. V. Klein, in Light Scattering in Solids, edited by M. Cardona (Springer-Verlag, Berlin, 1975), p. 147.

${ }^{30}$ P. Hawrylak, Ji-Wei Wu, and J.J. Quinn, Phys. Rev. B 32, 5169 (1985).

${ }^{31}$ M.S.-C. Luo, Shun Lien, Chuang, S. Schmitt-Rink, and A. Pinczuk, Phys. Rev. B 48, 11086 (1993).

${ }^{32}$ L. Wendler, V.M. Fomin, A.V. Chaplik, and A.O. Govorov, Phys. Rev. B 54, 4794 (1996).

${ }^{33}$ M. Pi, M. Barranco, A. Emperador, E. Lipparini, and Ll. Serra, Phys. Rev. B 57, 14783 (1998).

${ }^{34}$ M. Stopa, Phys. Rev. B 54, 13767 (1996).

${ }^{35}$ D.A. Broido, K. Kempa, and B. Bakshi, Phys. Rev. B 42, 11400 (1990).

${ }^{36}$ V. Gudmundsson and J.J. Palacios, Phys. Rev. B 52, 11266 (1995).

${ }^{37}$ M. Ferconi and G. Vignale, Phys. Rev. B 50, 14722 (1994).

${ }^{38}$ O. Steffers, U. Rössler, and M. Suhrke, Europhys. Lett. 42, 529 (1998).

${ }^{39}$ E. Lipparini, M. Barranco, A. Emperador, M. Pi, and Ll. Serra, Phys. Rev. B 60, 8734 (1999).

${ }^{40}$ Notice that we associate a + sign with $-L$ and a - sign with $+L$. This is because our choice of $B$ pointing towards $+z$ favors negative orbital angular momentum (see Fig. 6). Therefore, the $-L$ operator corresponds to an increase in absolute value of the negative total angular momentum, i.e., a + sign. Analogously, $+L$ corresponds to a decrease ( - sign). We have used the same convention for quantum rings, see A. Emperador, M. Barranco, E. Lipparini, M. Pi, and Ll. Serra, Phys. Rev. B 59, 15301 (1999).

${ }^{41}$ L. Brey, N.F. Johnson, and B.I. Halperin, Phys. Rev. B 40, 10647 (1989); P.A. Maksym and T. Chakraborty, Phys. Rev. Lett. 65, 108 (1990)

${ }^{42}$ A. Lorke, M. Fricke, B. T. Miller, M. Haslinger, J.P. Kotthaus, G. Medeiros-Ribeiro, and P.M. Petroff, IOP Conf. Proc. No. 155 (Institute of Physics, London, 1997), p. 803. 